\title{
Large $N$ expansion for frustrated and doped quantum antiferromagnets
}

\author{
Subir Sachdev and N. Read \\ Center for Theoretical Physics, P.O. Box 6666 \\ and \\ Department of Applied Physics, P.O. Box 2157 \\ Yale University, New Haven, CT 06511
}

\begin{abstract}
A large $N$ expansion technique, based on symplectic $(S p(N))$ symmetry, for frustrated magnetic systems is studied. The phase diagram of a square lattice, spin $S$, quantum antiferromagnet with first, second and third neighbor antiferromagnetic coupling (the $J_{1^{-}} J_{2^{-}} J_{3}$ model) is determined in the large- $N$ limit and consequences of fluctuations at finite $N$ for the quantum disordered phases are discussed. In addition to phases with long range magnetic order, two classes of disordered phases are found: (i) states similar to those in unfrustrated systems with commensurate, collinear spin correlations, confinement of spinons, and spin-Peierls or valence-bond-solid order controlled by the value of $2 S(\bmod 4)$ or $2 S(\bmod 2)$; (ii) states with incommensurate, coplanar spin correlations, and unconfined bosonic spin- $1 / 2$ spinon excitations. The occurrence of "order from disorder" at large $S$ is discussed. Neither chirally ordered nor spin nematic states are found. Initial results on superconductivity in the $t-J$ model at $N=\infty$ and zero temperature are also presented.

PACS Nos. 75.10.Jm, 75.50.Ee, 74.65.+n

September, 1990

International Journal of Modern Physics B 5, 219, (1991).
\end{abstract}




\section{Introduction}

Spurred by the discovery of high temperature superconductivity [1], the last few years have seen intense activity in the subject of two-dimensional quantum antiferromagnets. In particular, much attention has been focussed upon understanding the structure of quantum disordered phases of such antiferromagnets [2] and their possible relationship to high temperature superconductivity [3]. Large $N$ expansions [4] on unfrustrated antiferromagnets found columnar spin-Peierls order in the disordered state for half-integer spins [5, 6, 7]. "Chiral" [8] and "spin-nematic" 9] states are among the new structures that have been proposed as ground states of frustrated quantum antiferromagnets.

This paper presents details of a new systematic analytic technique for frustrated and doped antiferromagnets (AFMs) which has been proposed recently [10]. The method relies on a large $N$ expansion based upon models with symplectic symmetry $(S p(N))$. The method is applied in this paper to two models:

(i) A square lattice AFM with first, second and third neighbor antiferromagnetic coupling - the $J_{1^{-}} J_{2^{-}} J_{3}$ model. This model has been studied elsewhere by numerical [11, 12, seriesexpansion [13], and mean-field [14] methods; we will compare our results with these studies later. A particular strength of our approach is that it makes the connection between the structure of the known classically ordered phases and the quantum disordered phases especially clear. It will also clarify the appearance of "order-from-disorder" [15] from quantum fluctuations for models which are classically disordered. We will find new quantum disordered phases which have unconfined bosonic spin- $1 / 2$ spinon excitations.

(ii) The square lattice $t-J$ model. We will present initial results in the mean field $N=\infty$

limit at $T=0$. We find superconducting ground states similar in structure to those of Ref [16]. 
We begin by motivating the use of a symplectic large $N$ expansion for frustrated AFMs. Previous large $N$ methods have been based on the $S U(N)$ generalization of the $S U(2)$ AFM [4, 6, 7] and have been restricted to unfrustrated AFMs with a two sublattice structure (labeled $A, B)$ for reasons we now explore. "Spins" are placed on sublattice $A$ forming an irreducible representation of $S U(N)$ while those on $B$ form the conjugate representation. Thus e.g. totally symmetric representations on sublattice $A$ can be can be formed [4] by placing $n_{b}$ bosons created by $b_{i \alpha}^{\dagger}$ where $\alpha=1 \ldots N$ and $i \in A$; the $b^{\alpha}$ bosons transform under the fundamental representation of $S U(N)$. The conjugate representation is placed on sublattice $B$ by $n_{b}$ bosons created by $\bar{b}_{j}^{\dagger \alpha}$ for $j \in B$; the $\bar{b}_{\alpha}$ bosons transform under the representation conjugate to the fundamental. The use of conjugate representations ensures a natural pairing between directions in spin space on the two sublattices. The only possible coupling between sites on opposite sublattices which is invariant under $S U(N)$ and bilinear in the spin operators is

$$
-\left(b_{i \alpha}^{\dagger} \bar{b}_{j}^{\dagger \alpha}\right)\left(b_{i}^{\beta} \bar{b}_{j \beta}\right)
$$

(For $S U(2)$ this reduces to the usual $S_{i} \cdot S_{j}$ plus a constant). In the classical limit $\left(n_{b} \rightarrow \infty\right)$ this coupling will induce a Néel ground state

$$
\prod_{i \in A}\left(b_{i 1}^{\dagger}\right)^{n_{b}} \prod_{j \in B}\left(\bar{b}_{j}^{1 \dagger}\right)^{n_{b}}|0\rangle .
$$

In a system with strong quantum fluctuations, this coupling tries to maximize the number of valence bonds

$$
\left(b_{i \alpha}^{\dagger} \bar{b}_{j}^{\dagger \alpha}\right)|0\rangle
$$

between pairs of sites on opposite sublattices. The only bilinear coupling between sites on the same sublattice is the 'ferromagnetic' coupling

$$
-\left(b_{i \alpha}^{\dagger} b_{i^{\prime}}^{\alpha}\right)\left(b_{i^{\prime} \beta}^{\dagger} b_{i}^{\beta}\right)
$$


which demands that the spins on sites $i$ and $i^{\prime}$ point in the same direction. Reversing the sign of this term will favor states in which the spins point in any two unequal directions; this is quite different from the antiferromagnetic coupling which explicitly pairs directions in spin space. Interactions between sites on the same and opposite lattices are thus inequivalent and there is no natural way of introducing a democratic antiferromagnetic coupling between any two sites. Only for $S U(2)$ do the ferromagnetic and antiferromagnetic couplings above become equivalent.

A proper description of a frustrated AFM therefore requires that all the spins transform under the same representation of a group, and that two spins can combine to form a singlet. For $S U(2)$ we can write $\bar{b}_{j \alpha} \equiv \varepsilon_{\alpha \beta} b_{j}^{\beta}\left(\varepsilon^{\sigma \sigma^{\prime}}=-\varepsilon^{\sigma^{\prime} \sigma}\right.$ and $\left.\varepsilon^{\uparrow \downarrow}=1\right)$; the boson annihilation operators on all the sites now have an upper index. Singlet bonds $\varepsilon^{\sigma \sigma^{\prime}} b_{i \sigma}^{\dagger} b_{j \sigma^{\prime}}^{\dagger}$ can now be formed between any two sites. This structure has a natural generalization to the symplectic groups $S p(N)$ for all $N$. These are the groups of $2 N \times 2 N$ unitary matrices $U$ such that

$$
U^{T} \mathcal{J} U=\mathcal{J}
$$

where

$$
\mathcal{J}_{\alpha \beta}=\mathcal{J}^{\alpha \beta}=\left(\begin{array}{ccccc}
1 & & & \\
-1 & & & & \\
& & 1 & & \\
& -1 & & & \\
& & & \ddots & \\
& & & & \ddots
\end{array}\right)
$$

is the generalization of the $\varepsilon$ tensor (note $S p(1) \cong S U(2)$ ). This tensor can be used to raise or lower indices on other tensors; all representations are therefore self-conjugate. "Spins" can be created on each site by $n_{b}$ bosons $b_{i \alpha}^{\dagger}$ where $\alpha=1 \ldots 2 N$. The $b_{i}^{\alpha}$ bosons transform as the fundamental representation of $S p(N)$; the "spins" on the lattice therefore belong to the symmetric product of $n_{b}$ fundamentals, which is also an irreducible representation. Valence 
bonds

$$
\mathcal{J}^{\alpha \beta} b_{i \alpha}^{\dagger} b_{j \alpha}^{\dagger}
$$

can be formed between any two sites; this operator is a singlet under $S p(N)$ because of (15). An antiferromagnetic coupling should maximize the number of such bonds between two sites; we will therefore consider models described by the following Hamiltonian

$$
H_{A F}=-\sum_{i>j} \frac{J_{i j}}{N}\left(\mathcal{J}^{\alpha \beta} b_{i \alpha}^{\dagger} b_{j, \beta}^{\dagger}\right)\left(\mathcal{J}_{\gamma \delta} b_{i}^{\gamma} b_{j}^{\delta}\right)
$$

where $i, j$ run over the sites of an arbitrary lattice, and $J_{i j}$ are antiferromagnetic exchange constants. We recall the constraint

$$
b_{i \alpha}^{\dagger} b_{i}^{\alpha}=n_{b}
$$

which must be imposed at every site. For the group $S p(1)$ this generates states with spin $S=n_{b} / 2$ at every site.

We now summarize the results obtained in our study of $H_{A F}$ for the model with nearest $\left(J_{1}\right)$, second $\left(J_{2}\right)$ and third $\left(J_{3}\right)$ neighbor interactions on the square lattice. The large $N$ limit was taken with the ratio $n_{b} / N$ fixed; the results depend strongly on the value of $n_{b} / N$. The phases found (Figs 11, 2, 3, 4) can be separated into two distinct types:

\section{Commensurate, collinear phases}

These are closely related to those found in unfrustrated $S U(N)$ AFMs [6, 7]. For large values of $n_{b} / N$ these states have magnetic long range order (LRO) with the spins polarized parallel or anti-parallel to each other. Upon reducing $n_{b} / N$ a transition eventually occurs to a corresponding short range ordered (SRO) phase, with a finite spin-correlation length. All the SRO phases are described at long distances and long times by the following effective action when the correlation length is not too small:

$$
S_{e f f}=\int d^{2} r \int_{0}^{c \beta} d \tilde{\tau}\left\{\frac{1}{g}\left[\left|\left(\partial_{\mu}-i A_{\mu}\right) z^{\alpha}\right|^{2}+\frac{\Delta^{2}}{c^{2}}\left|z^{\alpha}\right|^{2}\right]\right\}+\cdots,
$$


where $\tau$ is the Matsubara time, $c$ is the spin-wave velocity, $\tilde{\tau}=c \tau$, and $\mu$ runs over $x, y, \tilde{\tau}$. Spatial anisotropy can be also be present but has been neglected for simplicity. The action describes a complex field $z^{\alpha}$ which transforms under the fundamental of $S p(N)$ and has a $U(1)$ charge +1 ; this field is related to the lattice boson $b^{\alpha}$, although the form of the relationship varies in different phases. The $U(1)$ gauge field $A_{\mu}$ is related to the phases of certain link-variables, and the gauge symmetry is compact. In the SRO phase, the mass $\Delta$ is finite and is proportional to the inverse spin-correlation length. The compact $U(1)$ gauge field leads to confinement of the $z$ quanta and Berry phases of its instantons (monopoles) [5] induce spin-Peierls order for special values of $n_{b}$ [7]. The LRO phase is reached when $\Delta$ vanishes and $z$ quanta condense in the $\mathbf{k}_{0}=0$ state [17. To characterize the LRO phases by a gauge-invariant order parameter, we consider the expectation value of the 'spin' operators $s_{a}=\left\langle z_{\alpha}^{*} S_{a \beta}^{\alpha} z^{\beta}\right\rangle\left(S_{a}\right.$ is a generator of $\left.S p(N)\right)$ across the transition. In the SRO phase these vanish as a result of $S p(N)$ invariance. In the LRO phase, the order parameter manifold, $M_{\text {coll }}$ will be given by $S p(N)$ modulo the subgroup which leave the $s_{a}$ invariant. Any orientation of the $z$ condensate can be rotated by $S p(N)$ transformations such that $\left\langle z^{\alpha}\right\rangle=\bar{z} \delta^{\alpha 1}$. Now the $s_{a}$ are invariant under the group $S p(N-1)$ acting on components $\alpha>2$. An additional factor of $U(1)$ is obtained from the symmetry associated with rotations about the ' $z$ ' axis in the $\alpha=1,2$ subspace under which $z^{1} \rightarrow z^{1} e^{i \phi}$ and $z^{2} \rightarrow z^{2} e^{-i \phi}$. We have therefore

$$
M_{\mathrm{coll}}=\frac{S p(N)}{U(1) \times S p(N-1)}
$$

in direct analogy with $U(N) /(U(1) \times U(N-1)) \cong C P^{N-1}$ for the $S U(N)$ models 6, 18; for $N=1$ we recover $C P^{1}$ (the unit sphere, $S^{2}$ ). The transition from LRO to SRO is expected to be described by a non-linear sigma $(N L \sigma)$ model on $M_{\text {coll }}$. We note that $\pi_{2}\left(M_{\text {coll }}\right)=Z$, the group of integers, so that topologically stable point defects in spacetime (hedgehogs) exist for all $N$ when the spatial dimension $d=2$, while $\pi_{1}=0$ so there are no line defects. 


\section{Incommensurate phases}

Frustration induces phases with LRO and SRO in incommensurate helical spin configurations. All of the phases found favor planar arrangement of spins and there is no "chiral" order. In regions not too far from a commensurate phase these states are described at long distances and long times by the following effective action:

$S_{\text {eff }}=\int d^{2} r \int_{0}^{c \beta} d \tilde{\tau}\left\{\frac{1}{g}\left[\left|\left(\partial_{\mu}-i A_{\mu}\right) z^{\alpha}\right|^{2}+\frac{\Delta^{2}}{c^{2}}\left|z^{\alpha}\right|^{2}\right]+\vec{\Phi} \cdot\left(\mathcal{J}_{\alpha \beta} z^{\alpha} \vec{\nabla} z^{\beta}\right)+\right.$ c.c. $\left.+V(\vec{\Phi})\right\}+\ldots$,

The new feature is the presence of a charge -2 two-component scalar $\vec{\Phi}=\left(\Phi_{x}, \Phi_{y}\right)$ related to lattice link fields which induce incommensurate order. The coupling between the $z$ and $\vec{\Phi}$ is the simplest one consistent with global $S p(N)$ symmetry and is $U(1)$ gauge invariant due to the antisymmetry of $\mathcal{J}$ (a coupling with no gradients of the form $\vec{\Phi} \mathcal{J}_{\alpha \beta} z^{\alpha} z^{\beta}$ vanishes identically). The potential $V(\vec{\Phi})$ is induced by short wavelength fluctuations; in the incommensurate phases $V(\vec{\Phi})$ will have minima at a non-zero value of $\vec{\Phi}$ leading to a Higgs phase with $\langle\vec{\Phi}\rangle \neq 0$. All of phases found in this paper have at least two gauge inequivalent minima related to each other by a square lattice symmetry; this leads to a two-fold degeneracy which can only be lifted by explicitly breaking the square lattice symmetry in $H_{A F}$. The minima are also such that $\left\langle\Phi_{x}\right\rangle$ and $\left\langle\Phi_{y}\right\rangle$ can simultaneously be made real in a suitable gauge. In such a gauge we see from Eqn (12) that the minima of the dispersion of the $z^{\alpha}$ quanta are at wavevectors $\mathbf{k}_{0}= \pm g\left(\left\langle\Phi_{x}\right\rangle,\left\langle\Phi_{y}\right\rangle\right) / 2$. For finite $\Delta$ this leads to incommensurate SRO. The $z^{\alpha}$ quanta have unit charge and will therefore be unconfined [19] in this Higgs phase: these SRO phases therefore possess unconfined bosonic spinons which transform under the fundamental of $S p(N)$ [20] for all values of the on-site 'spin' $n_{b}$. In models which have the two-fold degeneracy lifted by small explicit symmetry breaking terms in $H_{A F}$ these results appear to contradict the 'fractional quantization principle' of Laughlin [21]. As $\Delta \rightarrow 0$, the $z$ 
quanta will condense at $\mathbf{k}_{0}$ leading to incommensurate LRO. The order-parameter manifold is now

$$
M_{\text {noncoll }}=\frac{S p(N)}{Z_{2} \times S p(N-1)}
$$

because the $U(1)$ invariance about the ' $z$ ' axis has now been reduced to $Z_{2}$ as the spins are no longer collinear. This generalizes the result for $N=1, S O(3) \cong S U(2) / Z_{2}$ pointed out previously [22, 23]. For $M_{\text {noncoll }}, \pi_{2}=0$ but $\pi_{1}=Z_{2}$, so there are line defects in spacetime (vortex worldlines) for $d=2$ in this case. Instantons and vortices are suppressed in the Higgs phase, so their Berry phases are not expected to lead to spin-Peierls order. However the Berry phases could induce additional intermediate phases between the Higgs and confinement phases: this will be briefly discussed later.

The symplectic groups also have interesting applications to doped AFMs as described e.g. by a $t-J$ model. In such a model, the hopping term transfers spin from site to site and so resembles the ferromagnetic coupling (4). Thus in $S U(N)$ models with conjugate representations on neighboring sites there is no simple $S U(N)$-invariant hopping term for $N>2$; if instead one choses all sites with the same representation, hopping can be included but not antiferromagnetic exchange. Our symplectic approach allows inclusion of both exchange and hopping for all $N$. In particular, using fermions with $S p(N)$ indices for spins and bosons for holes, the large $N$ limit justifies the decoupling of Ref [16] and produces superconductivity; phase separation into an insulating AFM and hole-rich superconductor is also present. Details of this analysis at $N=\infty$ and zero temperature are presented in Section 4.

The outline of the rest of this paper is as follows. In Section 2 we introduce the general formalism of the $S p(N)$ large $N$ limit for frustrated AFMs. Section 3 presents detailed results on the $J_{1^{-}} J_{2^{-}} J_{3}$ model. Finally Section 4 discusses initial results on the $t$ - $J$ model. 


\section{Frustrated Antiferromagnets}

We begin by setting up the general framework for the large- $N$ expansion of antiferromagnets with Hamiltonian $H_{A F}($ Eqn (8) $)$. The large- $N$ limit is taken with $n_{b} / N$ fixed to an arbitrary value. Depending upon the values of the $J_{i j}$ and of $n_{b} / N$, the ground state of $H_{A F}$ may either break global $S p(N)$ symmetry and possess magnetic long-range-order (LRO) or be $S p(N)$ invariant with only short-range magnetic order (SRO). The structure of the large- $N$ limit is very similar to the $S U(N)$ case, and has been discussed extensively before [4, 7] for the SRO states. We will therefore present details mainly in the LRO phases. The large $N$ limit for these states is most conveniently taken by adapting the method of Brezin and Zinn-Justin [24]. We begin by introducing the parametrization

$$
b_{i}^{m \sigma} \equiv\left(\begin{array}{c}
\sqrt{N} x_{i}^{\sigma} \\
\tilde{b}_{i}^{\tilde{m} \sigma}
\end{array}\right)
$$

We have introduced a natural double-index notation $\alpha \equiv(m, \sigma)$ with $m=1 \ldots N$ and $\sigma=\uparrow, \downarrow$. The index $\tilde{m}=2, \ldots N$. The $x^{\sigma}$ field has been introduced to allow for a non-zero condensate $\left\langle b_{i}^{m \sigma}\right\rangle=\sqrt{N} \delta_{1}^{m} x_{i}^{\sigma}$; we will only consider models in which the condensate in the LRO phase can be transformed by a uniform global $S p(N)$ rotation into this form. We insert (14) into $H_{A F}$, decouple the quartic terms by Hubbard-Stratanovich fields $Q_{i j}$, and enforce the constraints by the Largrange multipliers $\lambda_{i}$. This yields

$$
\begin{gathered}
H_{M F}=\sum_{i>j}\left(N J_{i j}\left|Q_{i j}\right|^{2}-J_{i j} Q_{i j} \varepsilon_{\sigma \sigma^{\prime}}\left(N x_{i}^{\sigma} x_{j}^{\sigma \prime}+\sum_{\tilde{m}} \tilde{b}_{i}^{\tilde{m} \sigma} \tilde{b}_{j}^{\tilde{m} \sigma^{\prime}}\right)+\text { H. с. }\right) \\
+\sum_{i} \lambda_{i}\left(N\left|x_{i}^{\sigma}\right|^{2}+\sum_{\tilde{m}} \tilde{b}_{i, \tilde{m} \sigma}^{\dagger} \tilde{b}_{i}^{\tilde{m} \sigma}-n_{b}\right)
\end{gathered}
$$

The large $N$ limit is obtained by integrating over the $2(N-1) \tilde{b}$ fields. The resulting effective action, expressed in terms of the $Q_{i j}, \lambda_{i}$ and $x_{i}^{\sigma}$ fields, will have a prefactor of $N$ (and some terms of order 1 which are sub-dominant) and is therefore well approximated by its saddlepoint value. The $Q, \lambda, x$ fields are expected to be time-independent at the saddle-point 
and this is implicitly assumed in the following. The functional integral over the $\tilde{b}$ requires knowledge of the eigenmodes of $H_{M F}$. This can be done along standard lines: we first solve the eigenvalue equation

$$
\begin{aligned}
\lambda_{i} U_{i \mu}-\sum_{j} J_{i j} Q_{i j}^{*} V_{j \mu} & =\omega_{\mu} U_{i \mu} \\
\sum_{i} J_{i j} Q_{i j} U_{i \mu}-\lambda_{j} V_{j \mu} & =\omega_{\mu} V_{j \mu}
\end{aligned}
$$

for the $N_{s}$ (= number of sites in the system) positive eigenvalues $\omega_{\mu}$ and the corresponding eigenvectors $\left(U_{i \mu}, V_{j \mu}\right)$. The bosonic eigenoperators $\gamma_{\mu}^{\tilde{m} \sigma}$

$$
\gamma_{\mu}^{\tilde{m} \sigma}=\sum_{i}\left(U_{i \mu}^{*} \tilde{b}_{i}^{\tilde{m} \sigma}-\delta^{\tilde{m} \tilde{m}^{\prime}} \varepsilon^{\sigma \sigma^{\prime}} V_{i \mu}^{*} \tilde{b}_{i \tilde{m}^{\prime} \sigma^{\prime}}^{\dagger}\right)
$$

will diagonalize $H_{M F}$. The inverse relation is

$$
\tilde{b}_{i}^{\tilde{m} \sigma}=\sum_{\mu}\left(U_{i \mu} \gamma_{\mu}^{\tilde{m} \sigma}-\delta^{\tilde{m} \tilde{m}^{\prime}} \varepsilon^{\sigma \sigma^{\prime}} V_{i \mu}^{*} \gamma_{\mu \tilde{m}^{\prime} \sigma^{\prime}}^{\dagger}\right)
$$

Consistency of these relations imposes certain orthogonality requirements on $(U, V)$. It can be shown using Eqn (16) that these can always be satisfied. Finally the ground state energy, $E_{M F}$ of $H_{M F}$ is shown to be

$$
\frac{E_{M F}}{N}=\sum_{i>j}\left(J_{i j}\left|Q_{i j}\right|^{2}-J_{i j} Q_{i j} \varepsilon_{\sigma \sigma^{\prime}} x_{i}^{\sigma} x_{j}^{\sigma^{\prime}}+\text { H. c. }\right)-\sum_{i} \lambda_{i}\left(1+\frac{n_{b}}{N}-\left|x_{i}^{\sigma}\right|^{2}\right)+\sum_{\mu} \omega_{\mu}(Q, \lambda)
$$

In the last term we have emphasized that the $\omega_{\mu}$ depend upon $Q, \lambda$. Finding the ground state of $H_{A F}$ in the large $N$ limit is now reduced to the problem of minimizing $E_{M F}$ with respect to the independent variables $Q_{i j}, x_{i}^{\sigma}$ with the $\lambda_{i}$ chosen such that the constraints

$$
\frac{\partial E_{M F}}{\partial \lambda_{i}}=0
$$

are always satisfied. It is instructive to examine the equations obtained by demanding stationarity of $E_{M F}$ w.r.t $x_{i}^{\sigma}$ :

$$
\sum_{j} \varepsilon_{\sigma \sigma^{\prime}} J_{i j} Q_{i j} x_{j}^{\sigma^{\prime}}+\lambda_{i} x_{i \sigma}^{*}=0
$$


This equation has two possible solutions: (i) $x_{i}^{\sigma}=0$ : this gives the SRO phases (ii) $x_{i}^{\sigma} \neq 0$ : comparing Eqn (21) with Eqn (16) we see that this condition is equivalent to demanding that Eqn (16) possess at least one zero eigenvalue. For finite $n_{b}$, this will only occur in the limit $N_{s} \rightarrow \infty$, and implies the existence of gapless excitations. These are the Goldstone modes associated with the $S p(N)$ symmetry breaking.

We now examine the large $n_{b} / N$ limit of $H_{M F}$; it is easy to show that $Q_{i j} \sim \lambda_{i} \sim n_{b} / N$ while $x_{i}^{\sigma} \sim \sqrt{n_{b} / N}$. From Eqn (19) we observe that the sum over $\omega_{\mu}$ can be neglected and for large $n_{b} / N, E_{M F}$ reduces to

$$
\frac{E_{M F}^{c}}{N}=\sum_{i>j}\left(J_{i j}\left|Q_{i j}\right|^{2}-J_{i j} Q_{i j} \varepsilon_{\sigma \sigma^{\prime}} x_{i}^{\sigma} x_{j}^{\sigma^{\prime}}+\text { H. c. }\right)-\sum_{i} \lambda_{i}\left(\frac{n_{b}}{N}-\left|x_{i}^{\sigma}\right|^{2}\right)
$$

The minimization of $E_{M F}^{c}$ w.r.t. $Q_{i j}$ can now be easily carried out and the problem reduces to that of finding the classical ground state of $H_{A F}$. Thus the large $n_{b} / N$ limit of the present large $N$ equations is equivalent to the classical limit which involves $n_{b} \rightarrow \infty$ at fixed $N$. The fact that these two limits commute gives us further confidence on the usefulness of the present large $N$ procedure.

Finally we indicate how this formalism can be used to evaluate the spin-stiffness of the LRO phases in the large $N$ limit. One takes values of $x_{i}^{\sigma}$ which minimize $E_{M F}$ and performs a slowly varying rotation:

$$
x_{i}^{\sigma} \rightarrow\left(\exp \left(i \frac{\vec{n} \cdot \vec{\tau}}{2} \vec{k} \cdot \vec{R}_{i}\right)\right)_{\sigma \sigma^{\prime}} x_{i}^{\sigma^{\prime}}
$$

where $\vec{n}$ is the direction in spin-space about which the rotation has been performed, $\vec{\tau}$ are the Pauli matrices, and $\vec{k}$ is a small wavevector. We insert this value of $x_{i}^{\sigma}$ in $E_{M F}$, reminimize w.r.t. the $Q_{i j}$ while maintaining the constraints and determine the change in energy $\Delta E_{M F}$ for small $\vec{k}$. We expect in general

$$
\Delta E_{M F}=\frac{N_{s}}{2} \rho_{\alpha \beta}(\vec{n}) k_{\alpha} k_{\beta}+\cdots
$$


The co-efficient $\rho$ is the spin-stiffness tensor of the LRO phase about the direction $\vec{n}$.

\section{The $\mathrm{J}_{1}-\mathrm{J}_{2}-\mathrm{J}_{3}$ Model}

We now apply the formalism developed in the previous section to a frustrated antiferromagnet on the square lattice with first $\left(J_{1}\right)$, second $\left(J_{2}\right)$ and third $\left(J_{3}\right)$ neighbor interactions. This model has been examined by numerical [11, 12, series [13] and mean-field [14 methods in the literature; their results will be compared with ours in section 3.D. We begin by presenting the results of the $N=\infty$ mean field theory (section 3.A). The effective actions controlling the long-wavelength, long-time fluctuations at finite $N$ will be considered in section 3.B. Berry phases and the spin-Peierls order they induce will be disussed in section 3.C.

\section{A Mean Field Theory}

The energy $E_{M F}$ for this model was minimized over all fields $Q_{i j}$ and $\lambda_{i}$ which were periodic with a $\sqrt{2} \times \sqrt{2}$ unit cell. All of the global minima found in fact turned out to have a periodicity with one site per unit cell: we will therefore restrict our analysis to this simpler limit. In addition, full wavevector-dependent stability matrices involving quadratic fluctuations of $E_{M F}$ on the manifold specified by Eqn (20) were evaluated at representative points, and all eigenvalues found to be positive. This gives us reasonable confidence that the states described below are in fact the global minima of $E_{M F}$.

With one site per unit cell, the link variational parameters are the nearest neighbor fields $Q_{1, x}, Q_{1, y}$, the diagonal 2nd neighbor fields $Q_{2, y+x}, Q_{2, y-x}$ and the 3rd neighbor fields $Q_{3, x}, Q_{3, y}$. The bosonic eigenmodes can be calculated exactly and we find the eigenenergies:

$$
\begin{gathered}
\omega_{\mathbf{k}}=\left(\lambda^{2}-4\left|A_{\mathbf{k}}\right|^{2}\right)^{1 / 2} \\
A_{\mathbf{k}}=J_{1}\left(Q_{1, x} \sin k_{x}+Q_{1, y} \sin k_{y}\right)+J_{2}\left(Q_{2, y+x} \sin \left(k_{y}+k_{x}\right)+Q_{2, y-x} \sin \left(k_{y}-k_{x}\right)\right)
\end{gathered}
$$




$$
+J_{3}\left(Q_{3, x} \sin \left(2 k_{x}\right)+Q_{3, y} \sin \left(2 k_{y}\right)\right)
$$

The wavevector $\mathbf{k}$ extends over the first Brillouin zone of the square lattice.

Our results for $J_{3}=0$ are summarized in Fig प1. We begin by discussing the structure of the ground state at $N=\infty$. We find 3 distinct types of phases:

1. $(\pi, \pi)$

These states have $Q_{1, x}=Q_{1, y} \neq 0, Q_{2, y+x}=Q_{2, y-x}=Q_{3, x}=Q_{3, y}=0$ and are the analogs of the states found in $S U(N)$ systems. Inserting these values into Eqn (25) we see that $\omega_{\mathbf{k}}$ has its minima at $\pm(\pi / 2, \pi / 2)$ : this implies that the spin-spin correlation function, which involves the product of two bosonic pair correlation functions 4], will have a peak at $(\pi, \pi)$. We have a state with $x_{i}^{\sigma} \neq 0$ (LRO) for $N / n_{b}<2.5$ and the corresponding SRO state for $N / n_{b}>2.5$. The boundary between LRO and SRO is independent of $J_{2} / J_{1}$, but this is surely an artifact of the large $N$ limit. Finite $N$ fluctuations should be stronger as $J_{2} / J_{1}$ increases, causing the boundary to bend a little downwards to the right. While properties of these states can be calculated numerically for all values of $J_{2} / J_{1}$ and $N / n_{b}$, we tabulate a few analytic results for the LRO phase in an expansion in powers of $N / n_{b}$. The ground state energy is

$$
E_{M F}=-N N_{s}\left(\frac{n_{b}}{N}\right)^{2}\left[2 J_{1}+4\left(1-I_{1}\right) J_{1}\left(\frac{N}{n_{b}}\right)+\cdots\right]
$$

where $I_{1}=0.84205$ is obtained from an integral over the Brillouin zone. The spin-stiffness for spin rotations about an axis perpendicular to the Néel axis is found to be

$$
\rho_{\alpha \beta}=\delta_{\alpha \beta} N\left(\frac{n_{b}}{N}\right)^{2} \frac{1}{2}\left[J_{1}-2 J_{2}+\left[4 J_{2}\left(I_{2}-1\right)-J_{1}\left(I_{1}+I_{2}-2\right)\right]\left(\frac{N}{n_{b}}\right)+\cdots\right]
$$

with $I_{2}=1.39320$. 


\section{2. $(\pi, 0)$ or $(\mathbf{0}, \pi)$}

The $(0, \pi)$ states have $Q_{1, x}=0, Q_{1, y} \neq 0, Q_{2, y+x}=Q_{2, y-x} \neq 0$, and $Q_{3, x}=Q_{3, y}=0$ and occur with LRO and SRO. The degenerate $(\pi, 0)$ state is obtained with the mapping $x \leftrightarrow y$. For $J_{2} / J_{1}>1 / 2$, the classical limit, has independent Néel order on each of the $A$ and $B$ sublattices; quantum fluctuations, which are automatically included in the present approach, cause the Néel order parameters to align ("order from disorder") leading to the LRO states. To understand the nature of the transition between $\operatorname{LRO}$ at $(\pi, \pi)$ to $\operatorname{LRO}$ at $(\pi, 0)$ or $(0, \pi)$ we need the energy and stiffness of the latter states for small $N / n_{b}$. We find

$$
E_{M F}=-N N_{s}\left(\frac{n_{b}}{N}\right)^{2}\left[J_{1}+2 J_{2}+\left(2 J_{1}+4 J_{2}-4 J_{1} I_{3}\right)\left(\frac{N}{n_{b}}\right)+\cdots\right]
$$

where $I_{3}$ is a Brillouin zone integral whose value depends upon $J_{2} / J_{1}$; we will only need its value at $J_{2} / J_{1}=0.5: I_{3}=0.88241$. The stiffness of the $(0, \pi)$ state is soft at small $n_{b} / N$ for wavevectors in the $x$-direction:

$$
\rho_{x x}=N\left(\frac{n_{b}}{N}\right)^{2} \frac{1}{2}\left[2 J_{2}-J_{1}+2\left[J_{2}\left(2+I_{5}-2 I_{4}\right)+J_{1}\left(I_{4}-1\right)\right]\left(\frac{N}{n_{b}}\right)+\cdots\right]
$$

where $I_{4}, I_{5}$ are Brillouin zone integrals dependent upon $J_{2} / J_{1}$. Again we will only need their values at $J_{2} / J_{1}=0.5$ which are $I_{4}=1.28576$ and $I_{5}=0.32767$. Comparing Eqns (26) and (28) we see that the energies of the $(\pi, \pi)$-LRO and $(0, \pi)$-LRO states become equal at

$$
J_{2}=J_{1}\left[\frac{1}{2}+0.08072\left(\frac{N}{n_{b}}\right)+\cdots\right]
$$

At this value of $J_{2} / J_{1}$ the stiffness of the $(\pi, \pi)$ state is found to be

$$
\rho_{\alpha \beta}(\pi, \pi)=0.1949 N\left(\frac{n_{b}}{N}\right) \delta_{\alpha \beta}+\cdots
$$

while the stiffness of the $(0, \pi)$ state is

$$
\rho_{x x}(0, \pi)=0.2446 N\left(\frac{n_{b}}{N}\right)+\cdots
$$


Thus both stiffnesses are positive and indicate that the transition between the states is first order. Note that in both stiffnesses the leading $N\left(n_{b} / N\right)^{2}$ term is absent; these stiffnesses thus vanish classically and have appeared directly as a result of quantum fluctuationsanother example of "order from disorder". These results contradict arguments in Ref. [25] that another 'spin liquid' phase exists at arbitrarily small $N / n_{b}$ near $J_{2} / J_{1}=0.5$. While we agree that quantum fluctuations are very important in a 'fan' emanating from $J_{2} / J_{1}=0.5$, $N / n_{b}=0$, we have found that these fluctuations just reinforce the classical order. At larger values of $N / n_{b}$ we have a continuous (at $\left.N=\infty\right)$ transition to the $(0, \pi)$-SRO or $(\pi, 0)$-SRO state. The transition between this state and $(\pi, \pi)$-SRO remains first-order.

\section{3. "Decoupled"}

For $J_{2} / J_{1}$ and $N / n_{b}$ both large, we have a "decoupled" state with $Q_{2, y+x}=Q_{2, y-x} \neq 0$ and $Q_{1}=Q_{3}=0$. In this case $Q_{p}$ is non-zero only for sites on the same sublattice. The two sublattices have Néel type SRO which will be coupled by finite $N$ fluctuations. The $N=\infty$ state does not break any lattice symmetry.

We now turn to $J_{3} \neq 0$ (Figs 2, 3, 41), where we find a new class of phases:

\section{Incommensurate phases}

It is known that for finite $J_{3}$, the classical magnet has phases with incommensurate helical (coplanar) order [13]. Our phase diagram for a small value of $N / n_{b}\left(N / n_{b}=1\right.$, Fig [2a) is similar to the classical one. All of the LRO phases undergo continuous transitions to SRO with the same spatial distribution of the $Q_{p}$ and the accompanying broken rotational symmetry of the lattice. These SRO phases are shown in Fig 2 b. The two new classes of phases which did not appear at $J_{3}=0$ are: $(i) \pm(q, \pi)$ phases with $Q_{1, x} \neq Q_{1, y} \neq 0$, $Q_{2, x+y}=Q_{2, y-x} \neq 0, Q_{3, x} \neq 0$ and $Q_{3, y}=0 ;$ the degenerate $\pm(\pi, q)$ helix is obtained 
by the mapping $x \leftrightarrow y .(i i) \pm(q, q)$ phases with $Q_{1, x}=Q_{1, y} \neq 0, Q_{2, x+y} \neq 0, Q_{2, y-x}=0$, $Q_{3, x}=Q_{3, y} \neq 0$; this is degenerate with $(q,-q)$ phases [26]. The wavevector $q$ varies smoothly from 0 to $\pi$ and is determined by doubling the wavevector at which $\omega_{\mathbf{k}}$ has a minimum; it is continuous across second-order phase boundaries (Fig $2 \mathrm{a}$ ). In contrast to the classical limit [13] for $N / n_{b}$ finite it requires a finite $J_{3}$ to induce helical order; the first order transition from $(\pi, \pi)$ to $(\pi, 0)$ order persists for small $J_{3}$ as shown in the inset of Fig 2 . This suggests that quantum fluctuations induce an effective ferromagnetic $J_{3}$ in the model with bare $J_{3}=0$; a finite bare antiferromagnetic $J_{3}$ is required to compensate for this.

The broken discrete symmetries in states with $\mathrm{SRO}$ at $(0, \pi)$ and $(q, \pi)$ are identical: both are two-fold degenerate due to a breaking of the $x \leftrightarrow y$ symmetry. The states are only distinguished by a non-zero value of $Q_{3}$ in the $(q, \pi)$ phase and the accompanying incommensurate correlations in the spin-spin correlation functions. However $Q_{3}$ is gaugedependent and so somewhat unphysical as an order parameter. In the absence of any further fluctuation-driven lattice symmetry breaking (see below), the transition between SRO at $(0, \pi)$ and $(q, \pi)$ is an example of a disorder line [27; these are lines at which incommensurate correlations first turn on.

Fig 3 shows the transition from LRO to SRO as a function of $N / n_{b}$ for $J_{3}=0.35 J_{1}$. Similar transitions also appear in Fig 4 which has $J_{3}=J_{2} / 2$. There are several notable features of these two phase diagrams:

( $i$ ) An intermediate state in which $S p(N)$ symmetry is only partially restored (spin-nematic 9]) does not appear in the large $N$ limit. We expect the transition from $(q, q)$-LRO to $(q, q)$-SRO to be described by a $N L \sigma$ model on the manifold $M_{\text {noncoll }}$. A $d=1+\epsilon$ expansion of a similar $N L \sigma$ model 28] for $N=1$ has been recently carried out and does not show any tendency towards such ordering. 
(ii) The $Q_{p}$ variables can all be chosen real in all the phases, indicating the absence of "chiral" [8] order.

(iii) The commensurate states squeeze out the incommensurate phases as $N / n_{b}$ increases in both phase diagrams. We expect that this suppression of incommensurate order by quantum fluctuations is a general feature of frustrated AFMs.

\section{B Fluctuations - long wavelength effective actions}

This section will examine the form of the effective action controlling the long-wavelength fluctuations of the $b^{\alpha}$ quanta and the link fields $Q_{p}$. We will focus mainly on the SRO phases in the region close to the transition to the LRO phases.

We begin by examining the $(\pi, \pi)$-SRO phase; this phase has already been examined in considerable detail in Ref [7] for the $S U(N)$ AFM; here we adapt the results to the $S p(N)$ AFM. As noted above, this phase has the mean field values $Q_{1, x}=Q_{1, y}=\bar{Q}_{1}$, and $\omega_{\mathbf{k}}$ has minima at $\pm \vec{G}$, with $\vec{G}=(\pi / 2, \pi / 2)$. Close to the LRO phases, fluctuations of the $b^{\alpha}$ will be strong at $\pm \vec{G}$. We therefore parametrize

$$
b_{i}^{\alpha}=\varphi_{+}^{\alpha}\left(\vec{r}_{i}\right) e^{i \vec{G} \cdot \vec{r}_{i}}+\varphi_{-}^{\alpha}\left(\vec{r}_{i}\right) e^{-i \vec{G} \cdot \vec{r}_{i}}
$$

where $\varphi_{ \pm}$are assumed to have slow spatial variations. The most important component of the fluctuations of the $Q_{i j}$ are those of their phases. In particular, as noted in Ref [7], the mean-field theory has an unbroken gauge symmetry; the transformation

$$
b_{i}^{\alpha} \rightarrow b_{i}^{\alpha} e^{i \gamma_{i} \theta}
$$

where $\gamma_{i}=1(-1)$ for $r_{i x}+r_{i y}$ even (odd), leaves all $\left\langle Q_{i j}\right\rangle$ invariant. A staggered component of the phases of the $Q_{i j}$ acts as the vector potential associated with this gauge symmetry. We therefore write

$$
Q_{i, i+\hat{x}}=\bar{Q}_{1} \exp \left(i \gamma_{i} A_{x}\left(\vec{r}_{i}+\hat{x} / 2\right)\right)
$$


where again $A_{x}$ is slowly varying; a similar equation holds for $A_{y}$. The time-component of the gauge-field is the fluctuation in the Lagrange multiplier:

$$
\lambda_{i}=\bar{\lambda}+i \gamma_{i} A_{\tau}\left(\vec{r}_{i}\right)
$$

We now insert the ansatzes (33), (35) and (36) into the Lagrangian $\mathcal{L}$ associated with $H_{A F}$ and perform a gradient expansion on all the terms. The final result is most compactly expressed in terms of the fields

$$
\begin{aligned}
\psi_{1}^{\alpha} & =\left(\varphi_{+}^{\alpha}+\varphi_{-}^{\alpha}\right) / \sqrt{2} \\
\psi_{2 \alpha} & =-i \mathcal{J}_{\alpha \beta}\left(\varphi_{+}^{\beta}-\varphi_{-}^{\beta}\right) / \sqrt{2}
\end{aligned}
$$

and has the form

$$
\begin{gathered}
\mathcal{L}=\int \frac{d^{2} r}{a^{2}}\left[\psi_{1 \alpha}^{*}\left(\frac{d}{d \tau}+i A_{\tau}\right) \psi_{1}^{\alpha}+\psi_{2}^{\alpha *}\left(\frac{d}{d \tau}-i A_{\tau}\right) \psi_{2 \alpha}+\bar{\lambda}\left(\left|\psi_{1}^{\alpha}\right|^{2}+\left|\psi_{2 \alpha}\right|^{2}\right)\right. \\
\left.-4 J_{1} \bar{Q}_{1}\left(\psi_{1}^{\alpha} \psi_{2 \alpha}+\psi_{1 \alpha}^{*} \psi_{2}^{\alpha *}\right)+J_{1} \bar{Q}_{1} a^{2}\left[(\vec{\nabla}+i \vec{A}) \psi_{1}^{\alpha}(\vec{\nabla}-i \vec{A}) \psi_{2 \alpha}+(\vec{\nabla}-i \vec{A}) \psi_{1 \alpha}^{*}(\vec{\nabla}+i \vec{A}) \psi_{2}^{\alpha *}\right]\right]
\end{gathered}
$$

We now introduce the fields

$$
\begin{aligned}
z^{\alpha} & =\left(\psi_{1}^{\alpha}+\psi_{2}^{\alpha *}\right) / \sqrt{2} \\
\pi^{\alpha} & =\left(\psi_{1}^{\alpha}-\psi_{2}^{\alpha *}\right) / \sqrt{2}
\end{aligned}
$$

From Eqn (38), it is clear that the the $\pi$ fields turn out to have mass $\bar{\lambda}+4 J_{1} \bar{Q}_{1}$, while the $z$ fields have a mass $\bar{\lambda}-4 J_{1} \bar{Q}_{1}$ which vanishes at the transition to the LRO phase. The $\pi$ fields can therefore be safely integrated out, and $\mathcal{L}$ yields the following effective action, valid at distances much larger than the lattice spacing:

$$
S_{e f f}=\int \frac{d^{2} r}{\sqrt{8} a} \int_{0}^{c \beta} d \tilde{\tau}\left\{\left|\left(\partial_{\mu}-i A_{\mu}\right) z^{\alpha}\right|^{2}+\frac{\Delta^{2}}{c^{2}}\left|z^{\alpha}\right|^{2}\right\}
$$


Here $c=\sqrt{8} J_{1} \bar{Q}_{1} a$ is the spin-wave velocity, $\Delta=\left(\lambda^{2}-16 J_{1}^{2} \bar{Q}_{1}^{2}\right)^{1 / 2}$ is the gap towards spinon excitations, and $A_{\tilde{\tau}}=A_{\tau} / c$; this action is of the form (10) quoted in the introduction. Thus, in its final form, the long-wavelength theory consists of a massive $z^{\alpha}$ scalar (spinon) coupled to a compact $U(1)$ gauge field.

We now examine the changes in the above actions as one moves from the $(\pi, \pi)$-SRO phase into the $(q, q)$-SRO phase (Fig 2 $b$ ) (a very similar analysis can be performed at the boundary between the $(\pi, \pi)$-SRO and the $(\pi, q)$ - SRO phases). This transition is characterized by a continuous turning on of non-zero values of $Q_{i, i+\hat{y}+\hat{x}}, Q_{i, i+2 \hat{x}}$ and $Q_{i, i+2 \hat{y}}$. It is easy to see from Eqn (34) that these fields transform as scalars of charge $2 \gamma_{i}$ under gauge transformation associated with $A_{\mu}$. Performing a gradient expansion upon the bosonic fields coupled to these scalars we find that the Lagrangian $\mathcal{L}$ of the $(\pi, \pi)$-SRO phase gets modified to

$$
\mathcal{L} \rightarrow \mathcal{L}+\int \frac{d^{2} r}{a}\left(\vec{\Phi}_{A} \cdot\left(\mathcal{J}_{\alpha \beta} \psi_{1}^{\alpha} \vec{\nabla} \psi_{1}^{\beta}\right)+\vec{\Phi}_{B} \cdot\left(\mathcal{J}^{\alpha \beta} \psi_{2 \alpha} \vec{\nabla} \psi_{2 \beta}\right)+\text { c.c. }\right)
$$

where $\vec{\Phi}_{A, B} \equiv\left(J_{3} Q_{3, x}+J_{2} Q_{2, y+x}, J_{3} Q_{3, y}+J_{2} Q_{2, y+x}\right)$ with the sites on the ends of the link variables on sublattices $A, B$. Finally, as before, we transform to the $z, \pi$ variables, integrate out the $\pi$ fluctuations and obtain

$$
S_{\text {eff }}=\int \frac{d^{2} r}{\sqrt{8} a} \int_{0}^{c \beta} d \tilde{\tau}\left\{\left|\left(\partial_{\mu}-i A_{\mu}\right) z^{\alpha}\right|^{2}+\frac{\Delta^{2}}{c^{2}}\left|z^{\alpha}\right|^{2}+\vec{\Phi} \cdot\left(\mathcal{J}_{\alpha \beta} z^{\alpha} \vec{\nabla} z^{\beta}\right)+\text { c.c. }+V(\Phi)\right\}+\ldots
$$

Here $\vec{\Phi}=\left(\vec{\Phi}_{A}+\vec{\Phi}_{B}^{*}\right) /\left(2 J_{1} \bar{Q}_{1} a\right)$ is a scalar of charge -2 ; terms higher order in $\vec{\Phi}$ have been dropped. We have also added a phenomenological potential $V(\Phi)$ which is generated by short wavelength fluctuations of the $b^{\alpha}$ quanta. This action is of the general form (12), and as noted earlier, is the simplest one consistent with the requirements of $U(1)$-gauge and global $S p(N)$ invariance. In a phase with $\langle\vec{\Phi}\rangle \neq 0$ and real, we see from Eqn (41) that the minimum of the dispersion of the $z^{\alpha}$ quanta is at a wavevector $\mathbf{k}_{0}=\left(\left\langle\Phi_{x}\right\rangle,\left\langle\Phi_{y}\right\rangle\right) / 2$ : this will lead to 
incommensurate order. The action (41) thus demonstrates clearly the intimate connection between the Higgs phenomenon and the appearance of incommensurate correlations.

We now turn to the $(0, \pi)$-SRO phase; we will find that its properties are quite similar to the $(\pi, \pi)$-SRO phase. We recall that this phase has the mean-field values $Q_{2, y+x}=Q_{2, y-x}=$ $\bar{Q}_{2}, Q_{1, y}=\bar{Q}_{1}$ and $Q_{1, x}=0$. The excitation energies $\omega_{\mathbf{k}}$ now have minima at $\pm \vec{G}$ with $\vec{G}=(0, \pi / 2)$. We therefore parametrize the $b^{\alpha}$ fields as in Eqn (33), but using the new value of $\vec{G}$. This phase also has an unbroken $U(1)$ gauge symmetry; the main difference is in the staggering pattern of the charges of the $b^{\alpha}$ quanta. With the choice of the constants $\gamma_{i}=1(-1)$ for $r_{i y}$ even (odd) in Eqn (34) it is easily verified that all mean-field expectations $\left\langle Q_{i j}\right\rangle$ remain invariant. The phases of the link fields are parametrized as in Eqn (35) in terms of a vector potential $A$ but with the new $\gamma_{i}$; the diagonal link fields have the parametrization

$$
Q_{i, i+\hat{y}+\hat{x}}=\bar{Q}_{2} \exp \left[i \gamma_{i}\left(A_{y}\left(\vec{r}_{i}+\hat{y} / 2+\hat{x} / 2\right)+A_{x}\left(\vec{r}_{i}+\hat{y} / 2+\hat{x} / 2\right)\right)\right]
$$

and similarly for $Q_{i, i+\hat{y}-\hat{x}}$. The remaining analysis is essentially identical to that for the $(\pi, \pi)$-SRO phase and we obtain the same final effective action $S_{\text {eff }}$ (Eqn (39)) with an additional spatial anisotropy. The connection between the $z^{\alpha}$ quanta and the lattice bosons and between $A_{\mu}$ and the phases of the link fields are now of course different.

The transition from $(0, \pi)$-SRO to $(q, \pi)$-SRO follows the treatment above of the transition from $(\pi, \pi)$-SRO to $(q, q)$-SRO; the final action for the incommensurate phase has the same form as Eqn (41).

\section{C Berry Phases}

For the unfrustrated $S U(N)$ magnets, Berry phases associated with instanton tunneling events had particularly strong consequences in the SRO phase [5, 7]. Very similar effects occur in the commensurate SRO phases of the $J_{1^{-}} J_{2^{-}} J_{3}$ model. We consider the various 
phases in turn:

1. $(\pi, \pi)$

This phase is essentially identical to the $S U(N)$ case [7]. The instantons have Berry phases $n_{b} \zeta_{s} \pi / 2$ where $\zeta_{s}=0,1,2,3$ on dual lattice points with (even,even), (even,odd), (odd,odd), (odd, ,even) co- ordinates. Condensation of the instantons leads to spin-Peierls order of column (shown in Fig 1) or line type (not shown) for $n_{b}=1,3(\bmod 4)$ and 2 respectively, or a featureless VBS state [29] for $n_{b}=0(\bmod 4)$, throughout the $(\pi, \pi)$ SRO phase. The $b^{\alpha}$ quanta are confined by the compact $U(1)$ gauge force, with a confinement length scale determined by the instanton density [30].

\section{2. "Decoupled"}

We can apply the analysis of subsection 3.C.1 to each sublattice separately, giving e.g. for $n_{b}=1(\bmod 4)$ the type of spin-Peierls correlations shown in Fig 1. There is a total of $4 \times 4=16$ states for this case but coupling between the sublattices will reduce this to 8 states, all of one of the two types shown. The state with the 'dimers' parallel to one another has more possibilities for resonance using the $J_{1}$ bonds and is likely to be the ground state. For $n_{b}=2(\bmod 4)$, there will be $2 \times 2 / 2=2$ states, and for $n_{b}=0(\bmod 4)$, just one.

3. $(0, \pi)$

As in the other collinear phases, this state possesses instantons which are the remnants of hedgehogs in the LRO phase. A natural location for these instantons is at the center of the horizontal links which have $Q_{1, x}=0$; the spins at the ends of these links are ferromagnetically aligned and are most susceptible to large deformations. The Berry phase for a configuration of well separated instanton charges $m_{s}$ at co-ordinates $\mathbf{R}_{s}$ can be calculated as before [7, 5] 
and we find

$$
S_{B}=i n_{b} \sum_{s} \sum_{i}\left[m_{s} \gamma_{i} \theta_{s}\left(\mathbf{r}_{i}\right)\right]
$$

where $\theta_{s}\left(\mathbf{r}_{i}\right)$ is an angle which winds by $2 \pi$ as $\mathbf{r}_{i}$ moves around $\mathbf{R}_{s}$, the sum over $i$ extends over all the sites of the lattice and $\gamma_{i}$ is the staggering associated with the $(0, \pi)$ phase i.e. $\gamma_{i}=1(-1)$ for $r_{i y}$ even (odd). The sum over $i$ can be evaluated in a manner similar to Ref [5]. Consider first an isolated instanton of unit charge at $\mathbf{R}_{s}$; rewrite the summation in (43)) as one over vertical links of the square lattice:

$$
S_{B}=i n_{b} \sum_{i} \frac{\gamma_{i}}{2}\left[\theta_{s}\left(\mathbf{r}_{i}\right)-\theta_{s}\left(\mathbf{r}_{i+\hat{y}}\right)\right]
$$

The symmetry of the mean-field $(0, \pi)$ state with one instanton now requires that the contribution of the links be odd under reflection across the horizontal line running through $\mathbf{R}_{s}$. The majority of the links will cancel against their reflection partners, the only exceptions being the links which intersect the cut in the angle field $\theta_{s}\left(\mathbf{r}_{i}\right)$; these will yield an additional contribution of $i \pi n_{b}$. We expect that these symmetry considerations will also be valid for a configuration of well separated instantons. Combining the contributions of all the instantons we obtain finally

$$
S_{B}=i \pi n_{b} \sum_{s} m_{s}\left[R_{s x}\right]
$$

where $\left[R_{s x}\right]$ is the integer part of $R_{x}$. In the SRO phase the instantons interact with a Coulombic $1 / R$ potential; the instanton plasma can therefore be mapped onto a dual sineGordon model [7] in which the instanton Berry phases appear as frustrating phase-shifts in the arguments of the cosine term [7]. This model can be analyzed in a manner which closely parallels Ref [7]. The Berry phases lead to condensation of the instanton charges and to spinPeierls order of the type shown in Fig 1 for $n_{b}$ odd, and a VBS state for $n_{b}$ even. Combined with the choice $(0, \pi)$ or $(\pi, 0)$ this gives degeneracies $2,4,2,4$ for $n_{b}=0,1,2,3(\bmod 4)$. 


\section{Incommensurate Phases}

As the action (41) makes clear, these phases require an additional charge -2 field $\vec{\Phi}$ for their description. In the SRO phases, the $z^{\alpha}$ quanta can be integrated out and we are left with a lattice gauge theory of a compact $U(1)$ gauge field and a charge -2 scalar. The scalar fields can form vortices with flux quantum $\pi$ : instantons can change this flux by $2 \pi$, so only a $Z_{2}$ quantum number is topologically stable [31, in agreement with the analysis of the LRO phase. If the vortices proliferate it will be necessary to consider Berry phases associated with them and the instantons to understand the SRO phase. The following phases can occur in such a lattice gauge theory [19]:

( $i)$ A Higgs phase in which the vortices and instantons are suppressed; Berry phase effects are therefore unimportant and this phase will be insensitive to the precise value of $n_{b}$. The $b^{\alpha}$ quanta carry charge 1 and are unconfined [19]: these are spinons transforming under the fundamental of $S p(N)$. No such excitations were found in the commensurate phases. Gauge excitations have a gap controlled by the magnitude of the Higgs field $\langle\vec{\Phi}\rangle$. The incommensuration is also controlled by $\langle\vec{\Phi}\rangle$, as has been noted earlier. There is no breaking of lattice symmetries beyond those found at $N=\infty$. However with periodic boundary conditions the ground state has an additional factor of 4 degeneracy for all $n_{b}$ : the additional states are obtained by changing the sign of all $Q_{p}$ fields cut by a loop wrapped around the system [31]. This phase is expected to survive in our phase diagram at finite $N$.

(ii) A confinement phase with proliferation of vortices and instantons, confinement of spinons, and a gap towards gauge excitations controlled by the instanton density [30]. A plausible scenario is that this phase in fact coincides with the $(\pi, \pi)$-SRO phase and possesses spinPeierls order driven by the Berry phases of instantons.

(iii) Additional intermediate phases driven by the Berry phases of the instantons and 
vortices - this possibility is under investigation.

\section{D Comparison with numerical and series results}

Many numerical [1] and series analyses [13] have appeared on the model with $J_{3}=0$ for the spin-1/2 SU(2) model i.e. $N=1, n_{b}=1$ in the notation of this paper. They find $(\pi, \pi)$ LRO at small $J_{2} / J_{1},(\pi, 0)$-LRO at large $J_{2} / J_{1}$ and an intermediate SRO phase around $J_{2} / J_{1}=1 / 2$. This is in agreement (see Fig 10) with our prediction in section 3.A.1 that the phase boundary between $(\pi, \pi)$-LRO and $(\pi, \pi)$ - SRO bends downwards at finite $N$ with increasing $J_{2} / J_{1}$. Analyses of this intermediate phase at $J_{2} / J_{1}$ [11, 13] shows clear evidence of columnar spin-Peierls ordering [7], also in agreement with the results of Ref [7] and section 3.C.1. An additional intermediate phase with $(0, \pi)$-SRO has not been ruled out.

It would clearly be interesting to find the new incommensurate SRO phases with unconfined spinons of this paper in numerical work on models with $N=1, n_{b}=1$. Our results suggest that such phases will only occur in models with a finite $J_{3}$. A numerical analysis of the $J_{1^{-}} J_{2^{-}} J_{3}$ model along the line $J_{3}=0.5 J_{2}$ has recently been performed [12]. For comparison, we display the large $N$ results for these models in Fig 4 , As in Fig 4, these investigators 12 find correlations at $(\pi, \pi)$ at small $J_{2} / J_{1}$ and at $(0, \pi)$ at large $J_{2} / J_{1}$. Their results at intermediate $J_{2} / J_{1}$ are inconclusive but suggestive of a $(\pi, q)$ phase. A systematic analysis to resolve this issue and to distinguish SRO from LRO would be useful.

\section{The t-J Model}

This section will extend our results to a limited class of doped AFMs. These are described by the $t-J$ models in which electrons hop with matrix element $t$, have a nearest-neighbor antiferromagnetic exchange $J$, and have restrictions on their occupation number at each site 
due to the strong Coulomb interactions. Ideally one would like to dope AFMs which have spins in the representations examined above, i.e. transforming under the symmetric product of $n_{b}$ fundamentals of $S p(N)$, and take the large $N$ limit with $n_{b} / N$ constant. Parameters can then be chosen to obtain the experimentally observed [2] Néel LRO state in the undoped limit. These models require the introduction of bosons $b^{\alpha}$ carrying $S p(N)$ spin, and spinless fermions to represent the holes [26]. The Pauli exclusion principle now restricts the density of fermions to be order 1 and it appears that a simple large- $N$ limit does not exist.

We examine here the doping of AFMs which have spins transforming under the antisymmetric product of $m$ fundamentals of $S p(N)$ (this representation is not irreducible); the large $N$ limit will be taken with $m / N$ constant. Such spins are most conveniently described by introducing fermions $f^{\alpha}$ transforming under the fundamental of $S p(N)$. The holes are now represented by spinless bosons $b$. The physical 'electron' is therefore

$$
c_{i}^{\alpha}=f_{i}^{\alpha} b_{i}^{\dagger}
$$

The local constraint of the $t-J$ model is

$$
f_{i \alpha}^{\dagger} f_{i}^{\alpha}+b_{i}^{\dagger} b_{i}=m
$$

for every site $i$. We will focus exclusively on the case in which the states are half filled at zero doping: $m=N$. This approach is an adaptation of the large $N$ limits of Ref [32, 33] for $S U(N) t-J$ models. Unlike the $S U(N)$ case, the states on each site do not form an irreducible representation of $S p(N)$ at zero doping. Two fermions can combine to form on-site singlets $\mathcal{J}^{\alpha \beta} f_{i \alpha}^{\dagger} f_{i \beta}^{\dagger}$. There is, however, no energy gained out of forming such states; they in fact reduce the ability of the site to form energetically favorable singlet bonds with other sites.

For undoped AFMs with exchange interactions of the type (8), the large $N$ limit can be taken following the $S U(N)$ case. For weak frustration 34] the ground states are fully 
dimerized into pairs of sites forming singlet bonds. (Note that the bosonic large $N$ theories of Section 3 also give only dimerized ground states in the limit of large $N / n_{b}$ for $n_{b}$ odd: see Figs 1, 3, 4.) Néel -type states which are important for $N=1$ are not found. However at large dopings, the present large- $N$ offers a natural way of describing metallic states with a Fermi surface satisfying Luttinger's theorem, the existence of which appears to be suggested by some experiments on the high- $T_{c}$ materials [35. Thus the following calculation, while probably not experimentally relevant at small doping, might capture the correct physics at moderate and large dopings.

We will consider the following Hamiltonian

$$
\begin{array}{r}
H_{t J}=-\frac{t}{N} \sum_{<i j>} b_{i} f_{i \alpha}^{\dagger} f_{j}^{\alpha} b_{j}^{\dagger}-\frac{J}{N} \sum_{<i j>}\left(\mathcal{J}^{\alpha \beta} f_{i \alpha}^{\dagger} f_{j \beta}^{\dagger}\right)\left(\mathcal{J}_{\gamma \delta} f_{j}^{\delta} f_{i}^{\gamma}\right) \\
+\sum_{i} \lambda_{i}\left(f_{i \alpha}^{\dagger} f_{i}^{\alpha}+b_{i}^{\dagger} b_{i}-N\right)+\mu \sum_{i}\left(b_{i}^{\dagger} b_{i}-N \delta\right)
\end{array}
$$

The last two terms enforce the local constraint (47) and fix the average hole density at $N \delta$. Here we will focus exclusively on the $N=\infty$ limit at zero temperature. This requires a complete condensation of the $b$ bosons $\left\langle b_{i}\right\rangle=\sqrt{N} \bar{b}_{i}$ and introduction of the link-field

$$
\Delta_{i j}=\frac{1}{N}\left\langle\mathcal{J}^{\alpha \beta} f_{i \alpha}^{\dagger} f_{j \beta}^{\dagger}\right\rangle
$$

which performs the Hubbard-Stratanovich decomposition of the exchange interaction. We note that this decoupling is very similar to that performed in Refs [16]; in the present calculation however it is uniquely enforced by the large $N$ limit. As in Ref [16], at $T=0$, our solutions have $\bar{b}_{i} \neq 0$ (for $\delta \neq 0$ ) and $\Delta_{i j} \neq 0$ which describe a superconducting state. We have examined mean-field equations numerically with two sites per unit cell (with both the $2 \times 1$ and $\sqrt{2} \times \sqrt{2}$ unit cells) and determined the global ground states over this manifold. The results are summarized in Fig 5. A large part of the phase diagram in fact turns out 
to have a unit cell of just one site. In this case $\bar{b}_{i}=\sqrt{\delta}, \Delta_{i, i+\hat{x}} \equiv \Delta_{x}, \Delta_{i, i+\hat{y}} \equiv \Delta_{y}$ and the quasi-particle energy spectrum $\epsilon_{\mathbf{k}}$ is

$$
\epsilon_{\mathbf{k}}=\left[\left(\lambda-2 t \delta\left(\cos k_{x}+\cos k_{y}\right)\right)^{2}+4 J^{2}\left|\Delta_{x} \cos k_{x}+\Delta_{y} \cos k_{y}\right|^{2}\right]^{1 / 2}
$$

We find 4 different types of superconducting states which are described below. With the exception of the first phase which has coexisting spin-Peierls order, all phases are combination of extended $s\left(s^{*}\right)$ and $d$ wave pairing with no on-site pairing.

( $i)$ Coexistence of spin-Peierls order and superconductivity: This phase occurs for small $t$ or small $\delta$ and has a $2 \times 1$ unit cell with the spatial distribution of the $\Delta_{i j}$ shown in Fig [5, All three $\Delta_{1}, \Delta_{2}, \Delta_{3}$ cannot be made real in any gauge so this phase also breaks time-reversal invariance. At $t=0$ and $\delta$ arbitrary, or $\delta=0$ and $t$ arbitrary, we have $\Delta_{1} \neq 0, \Delta_{2}=\Delta_{3}=0$ : the system is now a fully dimerized insulator. The quasi-particle spectrum is found to have a gap over the entire Brillouin zone. This is the only phase which has a unit cell larger than one site.

(ii) $s^{*}+i d$-wave superconductor: Occurs around $t / J \approx 1$ and $\delta \approx 0.5$ and has $\Delta_{x}=$ $e^{i \theta} \Delta_{y}$ where the phase $\theta$ varies smoothly with $t / J$ and $\delta$. Such a state has been considered previously by Kotliar [36]. The quasi-particle spectrum is fully gapped and time-reversal symmetry is broken.

(iii) $d$-wave superconductor: This phase occurs for moderate and large $t / J$ at small $\delta$ and has $\Delta_{x}=-\Delta_{y}$ and has been considered previously by Kotliar and Liu [37]. The quasi-particle gap vanishes at 4 isolated points $\left( \pm k^{0}, \pm k^{0}\right)$ in the Brilluoin zone.

(iv) $s^{*}$-wave superconductor: Finally for large $\delta$ we obtain an extended $s$-wave solution with no on-site pairing which has $\Delta_{x}=\Delta_{y}$. There is again a fully gapped excitation spectrum; for large $t \delta / J$, the gap vanishes as $t \delta \exp (-c t \delta / J)$ where $c$ is a constant of order unity.

Finally we address the issue of the stability of the phases discussed above towards phase 
separation. A realistic model of the high- $T_{c}$ materials should also include the long-range Coulomb interactions between the electrons and between the rare-earth ions and the electrons. These interactions will reduce any tendency towards phase separation. It is nevertheless interesting to examine this issue for $H_{t J}$ alone. The results of a Maxwell construction on the energy-versus-doping curve are shown in Fig 5 . The region below the dotted line is susceptible to separation into an insulating antiferromagnet with $\delta=0$ and a hole-rich phase with $\delta$ on the dotted line. The boundary towards phase separation approaches $\delta=0$ as $t / J \rightarrow \infty$, in agreement with $\operatorname{Ref}[38$. However at small values of $t / J$, in particular at $t=0$, we find no phase separation, unlike Emery et. al. 38. The absence of phase separation at $t=0$ in the present calculation is due to the extra stability acquired at large $N$ by fully dimerized solutions with every site less than half-filled.

\section{Conclusions}

This paper has presented details on the application of a new large $N$ expansion based on the symplectic groups $S p(N)$ which was introduced recently [10]. These groups naturally generalize to all values of $N$ the physics of $S U(2) \cong S p(1)$ antiferromagnetism: the pairing of spins with opposite directions in spin space. They lead to a particularly appealing treatment of frustrated antiferromagnets with all antiferromagnetic exchange interactions treated democratically. The large $N$ limit was taken by placing spins on each site which transform as $n_{b}$ symmetric products on the fundamental of $S p(N)$, and fixing $n_{b} / N$ at an arbitrary value $\left(n_{b}=2 S\right.$ for $\left.S U(2)\right)$. For large $n_{b} / N$ the ground states become identical to the classical

solutions and possess magnetic long range order (LRO). Upon reducing $n_{b} / N$ all the LRO states under continuous transition to short range ordered (SRO) states whose properties are closely connected to the LRO states. In particular for the square lattice model with first, 
second, and third neighbor interactions (Figs 1, 2, 3, 4) we found two distinct classes of states:

(i) LRO states with commensurate, collinear order led at smaller values of $n_{b} / N$ to SRO states with a gap to all excitations, spinons confined by a compact $U(1)$ gauge force, and spin-Peierls or valence-bond-solid order controlled by the value of $n_{b}(\bmod 4)$.

(ii) LRO states with incommensurate, coplanar order led at smaller values of $n_{b} / N$ to SRO states with a gap to all excitations, unconfined spinons and no spin-Peierls order. A charge -2 scalar in a Higgs phase was responsible for liberating the spinons [19]. These incommensurate SRO states appear to contradict the 'fractional quantization principle' of Laughlin [21]. This principle asserts that spin-1/2 excitations of a non-degenerate 'spin-liquid' ground state must interact with a gauge force which endows them with fractional statistics. The SRO states found in this paper have a two-fold degeneracy but this can easily be lifted by adding small explicit symmetry breaking terms to $H_{A F}$ (this can be done for the $(q, \pi)$-SRO phase by modifying $J_{3}$ on just the $x$-directed links, and for the $(q, q)$-SRO phase by changing $J_{2}$ on the links in the $(1,1)$ direction). The modified model now has a non-degenerate ground state, no gapless excitations, and unconfined spin-1/2 spinon excitations with bosonic statistics, contradicting Ref [21].

Additional intermediate phases between the Higgs and confinement SRO phases are possible and are presently under investigation. No chirally ordered [8] or spin-nematic [9] states were found.

An initial discussion of the application of the symplectic groups to doped antiferromagnets was also presented. In this case they allow a natural implementation of both antiferromagnetic exchange and hole hopping terms, and emphasize the natural connection between antiferromagnetism and singlet superconductive pairing [3]. In particular a study of the 
$T=0, N=\infty$ limit of a $t-J$ model with spins represented by fermions and holes by spinless bosons yields superconducting ground states (Fig [5 in much the same manner as Ref [16]. Extension of these results to finite temperature and finite $N$ is clearly of great interest and is currently in progress.

\section{Acknowledgments}

We thank D.P. Arovas, P. Chandra, P. Coleman, E. Dagotto, G. Kotliar, J.B. Marston, A. Moreo, R. Shankar, and Z. Wang for useful discussions. This work was supported by the A. P. Sloan Foundation (N.R. and S.S.) and by NSF PYI Grant DMR 8857228 (S.S.).

\section{References}

[1] J.G. Bednorz and K.A. Muller, Z. Phys. B64, 188 (1986); M.K. Wu et. al., Phys. Rev. Lett. 58, 908 (1987).

[2] S. Chakravarty, B.I. Halperin, and D.R. Nelson, Phys. Rev. Lett. 60, 1057 (1988), Phys. Rev. B 39, 2344 (1989).

[3] P.W. Anderson, Science 235, 1196 (1987); S. Liang, B. Doucot, and P.W. Anderson, Phys. Rev. Lett. 61, 365 (1988).

[4] D.P. Arovas and A. Auerbach, Phys. Rev. B 38, 316 (1988); Phys. Rev. Lett. 61, 617 (1988).

[5] F.D.M. Haldane, Phys. Rev. Lett. 61, 1029 (1988)

[6] N. Read and S. Sachdev, Nucl. Phys. B316, 609 (1989).

[7] N. Read and S. Sachdev, Phys. Rev. Lett. 62, 1694 (1989); Phys. Rev. B (in press). 
[8] V. Kalmeyer and R.B. Laughlin, Phys. Rev. Lett. 59, 2095 (1987). X.-G. Wen, F. Wilczek and A. Zee, Phys. Rev. B 39, 11413 (1989).

[9] P. Chandra, P. Coleman, and A.I. Larkin, Phys. Rev. Lett. 64, 88 (1990); preprint; P. Chandra and P. Coleman, Int. J. Mod. Phys. B 3, 1729 (1989).

[10] N. Read and S. Sachdev, Phys. Rev. Lett. 66, 1773 (1991).

[11] E. Dagotto and A. Moreo, Phys. Rev. Lett. 632148 (1989); R.R.P. Singh and R. Narayan, Phys. Rev. Lett. 65, 1072 (1990).

[12] A. Moreo, E. Dagotto, T. Jolicoeur, and J. Riera, Phys. Rev. B 42, 6283 (1990).

[13] M.P. Gelfand, R.R.P. Singh, and D.A. Huse, Phys. Rev. B 40, 10801 (1989); M.P. Gelfand, preprint.

[14] S. Sachdev and R.N. Bhatt, Phys. Rev. B , 41, 4502 (1990).

[15] C. Henley, Phys. Rev. Lett. 62, 2056 (1989).

[16] G. Baskaran, Z. Zou, and P.W. Anderson, Sol. State Comm. 63, 973 (1987); A.E. Ruckenstein, P.J. Hirschfeld, and J. Appel, Phys. Rev. B 36, 857 (1987).

[17] D. Yoshioka, J. Phys. Soc. Jpn, 58, 3733 (1989).

[18] I. Affleck, Nucl. Phys. B257, 397 (1985); Nucl. Phys. B265, 409 (1985).

[19] See e.g. E. Fradkin and S.H. Shenker, Phys. Rev. D19, 3682 (1979).

[20] Unconfined spin-1/2 spinons are also predicted to appear in unfrustrated systems in $d=3 ;$ A. Kol and N. Read, unpublished.

[21] R.B. Laughlin, Science 242, 525 (1988). 
[22] B.I. Halperin and W.M. Saslow, Phys. Rev. B 16, 2154 (1977).

[23] T. Dombre and N. Read, Phys. Rev. B 39, 6797 (1989).

[24] E. Brezin and J. Zinn-Justin, Phys. Rev. B 14, 3110 (1976).

[25] P. Chandra and B. Doucot, Phys. Rev. B 38, 9335 (1988).

[26] Similar incommensurate states with valence bonds connecting sites on the same sublattice are also found in a study of doped systems; B.I. Shraiman and E.D. Siggia, Phys. Rev. Lett. 62, 1564 (1989); C. Jayaprakash, H.R. Krishnamurthy and S. Sarkar, Phys. Rev. B 40, 2610 (1989); C.L.Kane et al, ibid 41,2653 (1990); B. Chakraborty et al, Phys. Rev. B (in press).

[27] J. Stephenson, Can. J. Phys. 48, 2118, 1724 (1970).

[28] P. Azaria, B. Delamotte, and T. Jolicoeur, Phys. Rev. Lett. 64, 3175 (1990).

[29] I. Affleck, T. Kennedy, E.H. Lieb, and H. Tasaki, Phys. Rev. Lett. 59, 799 (1987); Commun. Math. Phys. 115, 477 (1988).

[30] A.M. Polyakov, Nucl. Phys. B 120, 429 (1977).

[31] N. Read and B. Chakraborty, Phys. Rev. B 40, 7133 (1989).

[32] I. Affleck and J.B. Marston, Phys. Rev. B37, 3774 (1988).

[33] M. Grilli and B.G. Kotliar, Phys. Rev. Lett. 64, 1170 (1990)

[34] D. Rokhsar, Phys. Rev. B 42, 2526 (1990) has shown that a large class of frustrated AFMs dimerize in the fermionic large- $N$ limit for $S U(N)$. It is likely that a similar result holds for the fermionic large- $N$ limit of $S p(N)$. 
[35] C. G. Olson et. al., Science 245, 731 (1989).

[36] B.G. Kotliar, Phys. Rev. B , 37, 3664 (1988).

[37] B.G. Kotliar and J. Liu Phys. Rev. B , 38, 5142 (1988).

[38] V.J. Emery, S.A. Kivelson, and H.Q. Lin, Phys. Rev. Lett. 64, 475 (1990). 


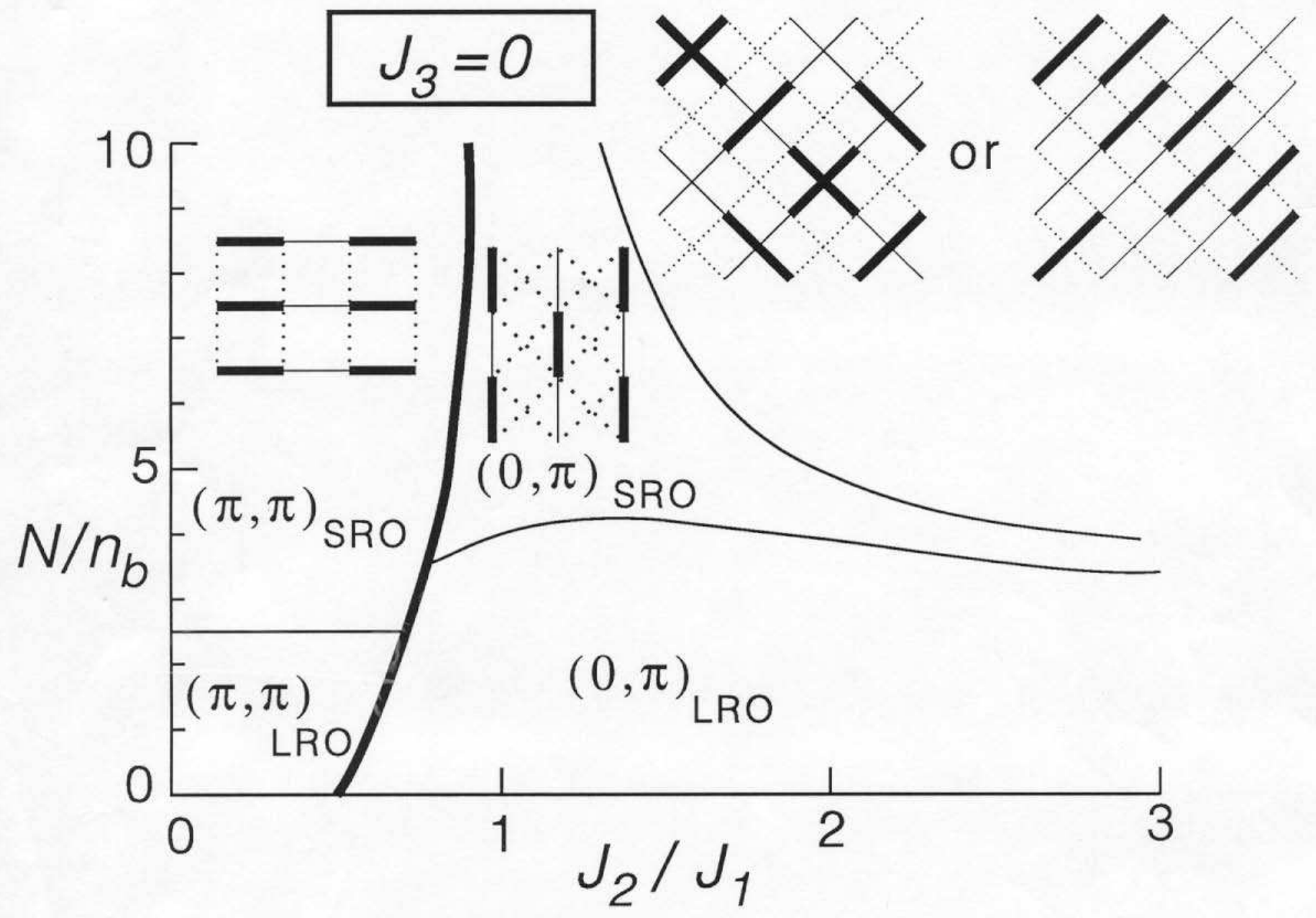

Figure 1: Ground states of $H$ for $J_{3}=0$ as a function of $J_{2} / J_{1}$ and $N / n_{b}\left(n_{b}=2 S\right.$ for $S p(1) \equiv S U(2)$ ). Thick (thin) lines denote first (second) order transitions at $N=\infty$. Phases are identified by the wavevectors at which they have magnetic long-range-order (LRO) or short-range-order (SRO). The links with $Q_{p} \neq 0$ in each SRO phase are shown. The large $N / n_{b}$, large $J_{2} / J_{1}$ phase has the two sublattices decoupled at $N=\infty$; each sublattice has Néel -type SRO. Spin-Peierls order at finite $N$ for odd $n_{b}$ is illustrated by the thick, thin and dotted lines. The $(\pi, \pi)$-SRO and the "decoupled" states have line-type [7] spin-Peierls order for $n_{b}=2(\bmod 4)$ and are VBS for $n_{b}=0(\bmod 4)$. The $(0, \pi)-$ SRO state is a VBS for all even $n_{b}$. 


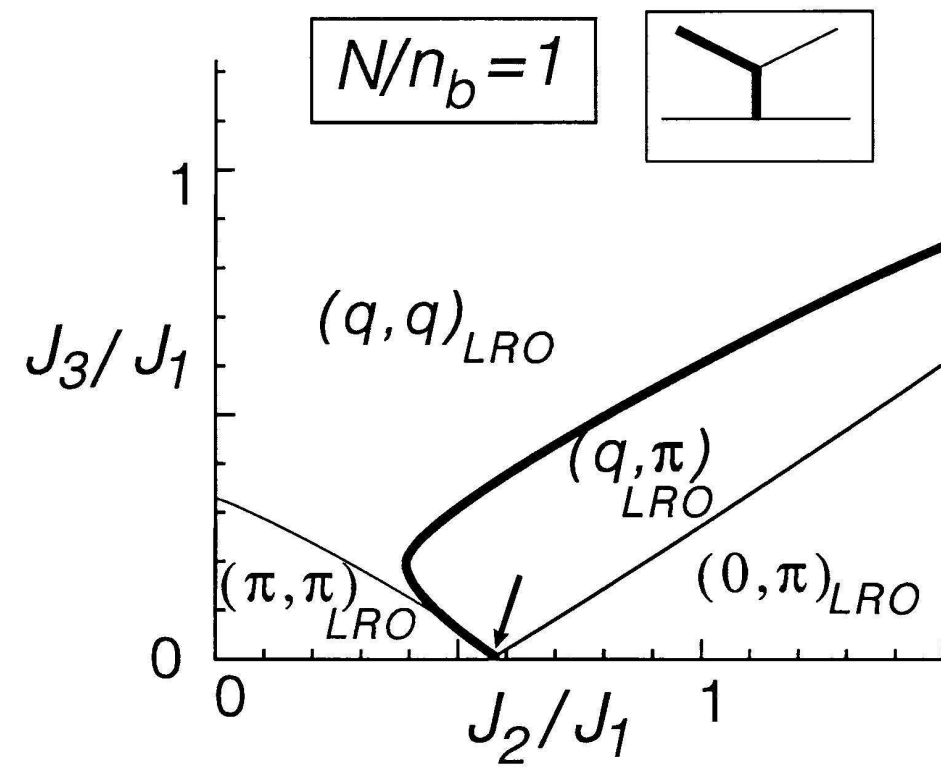

(a)

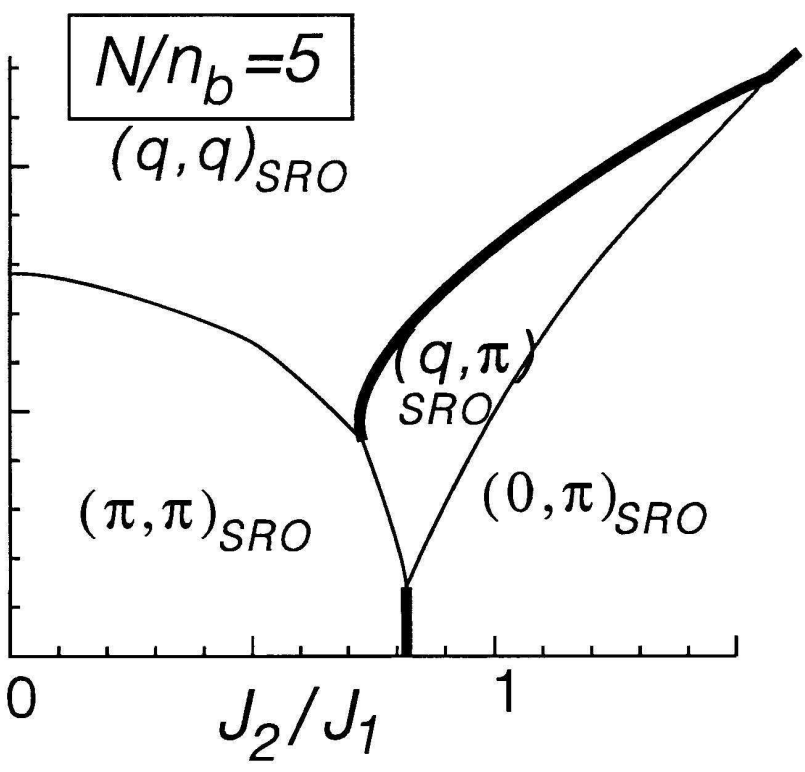

(b)

Figure 2: As in Fig 1 but as a function of $J_{2} / J_{1}$ and $J_{3} / J_{1}$ for $(a) N / n_{b}=1$ and $(b)$ $N / n_{b}=5$. The inset in $(a)$ shows the region at the tip of the arrow magnified by 20: a direct first-order transition from $(\pi, \pi)$-LRO to $(0, \pi)$-LRO occurs up to $J_{3} / J_{1}=0.005$. 


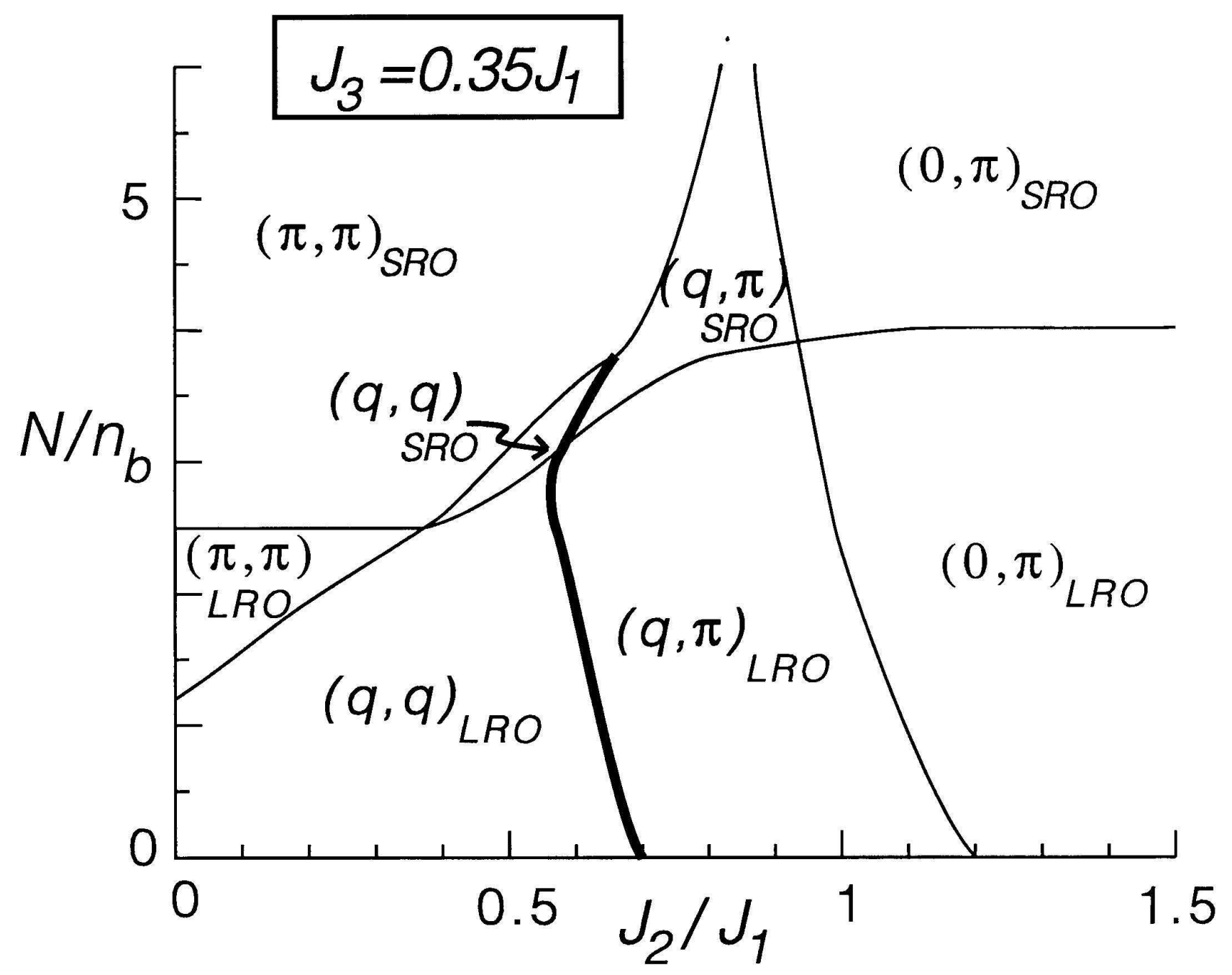

Figure 3: As in Fig 囵 but for $J_{3} / J_{1}=0.35$. 


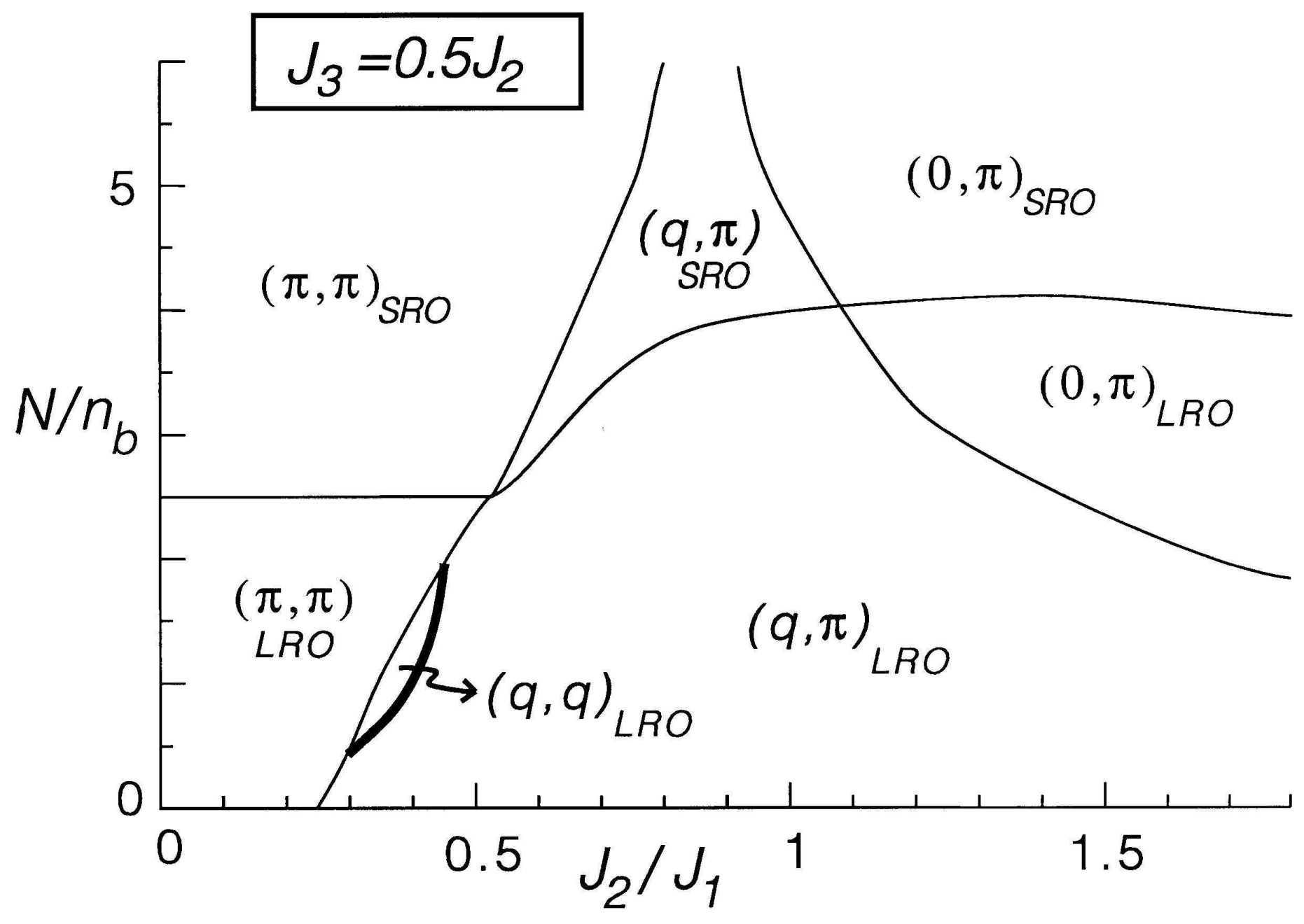

Figure 4: As in Fig प but for $J_{3} / J_{2}=0.5$. 


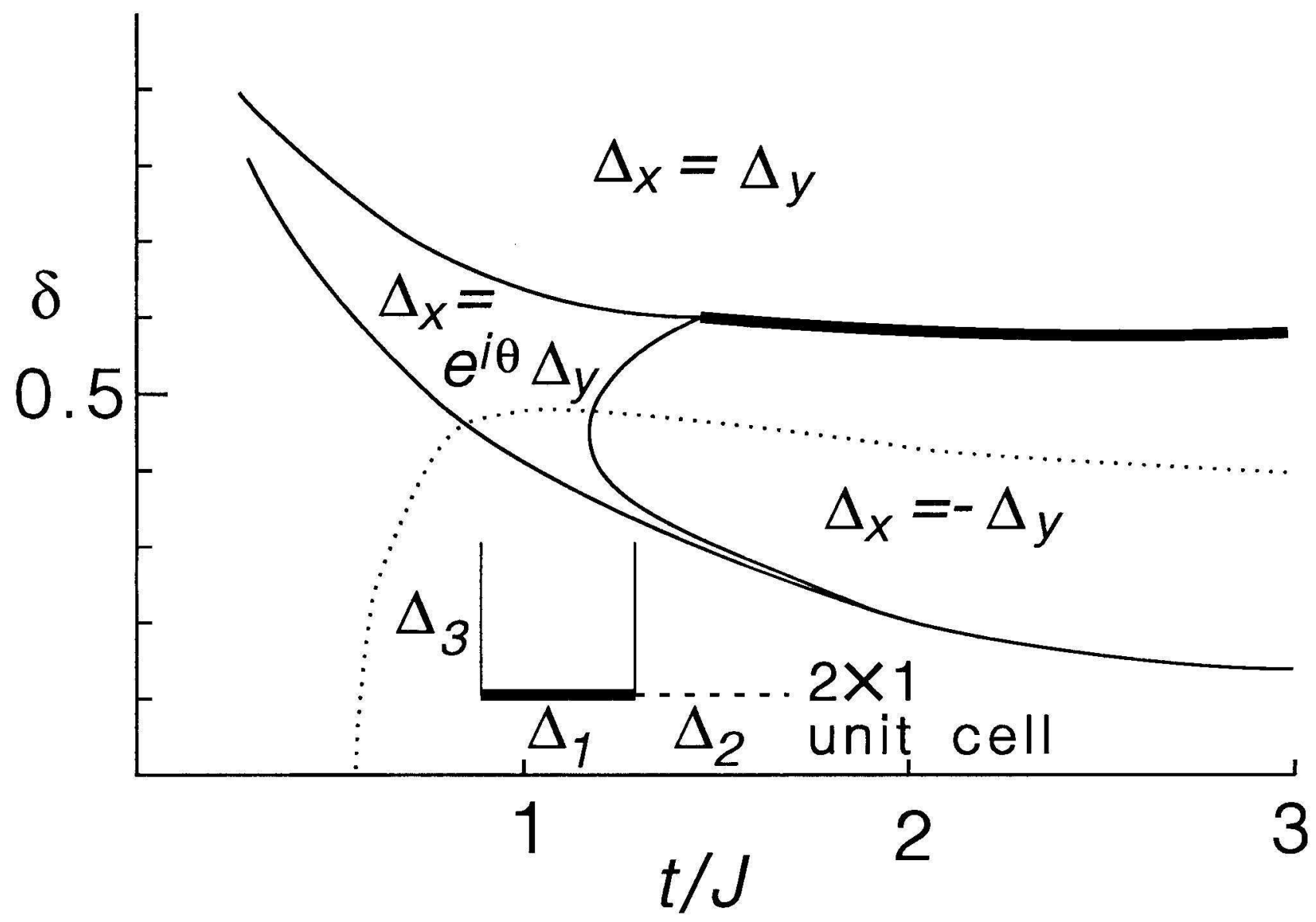

Figure 5: Ground state of the $t-J$ model $H_{t J}$ at $N=\infty$ as a function of $t / J$ and the hole concentration $\delta$. All phases except on the lines $t=0$ and $\delta=0$ are superconducting. The region below the dotted line is susceptible to separation into an insulating antiferromagnet with $\delta=0$ and a hole-rich phase with $\delta$ on the dotted line. The boundary towards phase separation approaches $\delta=0$ as $t / J \rightarrow \infty$ 